Edith Cowan University

Research Online

Research outputs 2012

$1-1-2012$

\title{
Effects of two contrast training programs on jump performance in rugby union players during a competition phase
}

\author{
C K Argus \\ N D Gill \\ J W Keogh \\ Michael Mcguigan \\ Edith Cowan University \\ W G Hopkins
}

Follow this and additional works at: https://ro.ecu.edu.au/ecuworks2012

Part of the Sports Sciences Commons, and the Sports Studies Commons

10.1123/ijspp.7.1.68

This is an Author's Accepted Manuscript of: Argus, C., Gill, N., Keogh, J., Mcguigan, M. R., \& Hopkins, W. (2012).

Effects of two contrast training programs on jump performance in rugby union players during a competition phase. International Journal of Sports Physiology and Performance, 7(1), 68-75. Available here (C) Human Kinetics, Inc.

This Journal Article is posted at Research Online.

https://ro.ecu.edu.au/ecuworks2012/500 


\title{
Effects of Two Contrast Training Programs on Jump Performance in Rugby Union Players During a Competition Phase
}

\author{
Christos K. Argus, Nicholas D. Gill, Justin W. L. Keogh, \\ Michael R. McGuigan, and Will G. Hopkins
}

\begin{abstract}
Purpose: There is little literature comparing contrast training programs typically performed by team-sport athletes within a competitive phase. We compared the effects of two contrast training programs on a range of measures in high-level rugby union players during the competition season. Methods: The programs consisted of a higher volume-load (strength-power) or lower volume-load (speed-power) resistance training; each included a tapering of loading (higher force early in the week, higher velocity later in the week) and was performed twice a week for 4 wk. Eighteen players were assessed for peak power during a bodyweight countermovement jump (BWCMJ), bodyweight squat jump (BWSJ), $50 \mathrm{~kg}$ countermovement jump (50CMJ), $50 \mathrm{~kg}$ squat jump (50SJ), broad jump (BJ), and reactive strength index (RSI; jump height divided by contact time during a depth jump). Players were then randomized to either training group and were reassessed following the intervention. Inferences were based on uncertainty in outcomes relative to thresholds for standardized changes. Results: There were small between-group differences in favor of strength-power training for mean changes in the 50CMJ $(8 \% ; 90 \%$ confidence limits, $\pm 8 \%)$, 50SJ $(8 \% ; \pm 10 \%)$, and BJ $(2 \% ; \pm 3 \%)$. Differences between groups for BWCMJ, BWSJ, and reactive strength index were unclear. For most measures there were smaller individual differences in changes with strength-power training. Conclusion: Our findings suggest that high-level rugby union athletes should be exposed to higher volume-load contrast training which includes one heavy lifting session each week for larger and more uniform adaptation to occur in explosive power throughout a competitive phase of the season.
\end{abstract}

Keywords: athlete, power, resistance, strength

The level of power an athlete possesses has been shown to distinguish between among levels of athletic ability and as such, increasing an athlete's ability to produce power may improve sporting performance..$^{1}$ Improving power in well-trained team sport athletes, especially during the competition phase of the season, can be difficult to achieve. Baker ${ }^{2}$ reported a $1 \%$ decrease in lower

Christos K. Argus is with Sports Performance Research Institute New Zealand, AUT University, Auckland, New Zealand. Nicholas D. Gill is with Sports Performance Research Institute New Zealand, AUT University, Auckland, New Zealand. Justin W.L. Keogh is with Sports Performance Research Institute New Zealand, AUT University, Auckland, New Zealand, and with the Faculty of Health Sciences and Medicine, Bond University, Gold Coast, Australia. Michael R. McGuigan is with Sports Performance Research Institute New Zealand, AUT University, Auckland, New Zealand, and with the School of Exercise, Biomedical and Health Sciences, Edith Cowan University, Australia. Will G. Hopkins is with Sports Performance Research Institute New Zealand, AUT University, Auckland, New Zealand. body mean power throughout a 29 wk competition phase in professional and college aged rugby league players. While more recently, a 3\% decrease in lower body peak power was observed during a $13 \mathrm{wk}$ competition phase in professional rugby union players. ${ }^{3}$ Consequently, training methods that improve power in already well-trained athletes during the competitive phase of the season need to be identified.

Programming methods consisting of the combination of strength training (lower velocity / higher force) and power training (higher velocity / lower force) have been regularly reported to be superior to strength or power training in isolation. ${ }^{4,5}$ Combined resistance training is commonly referred to as either compound training (heavy resistance day alternated with a lighter resistance day), complex training (several sets of a heavy resistance exercise that are followed by sets of a lighter resistance exercise) or contrast training (alternating heavy and lighter exercises set for set). ${ }^{6}$ Previous authors have reported larger improvements following combined training when compared with high strength or high power training alone. ${ }^{4,5}$ It has been postulated that combined 
training provides broader neuromuscular adaptations resulting in greater transfer to a wider variety of performance variables. ${ }^{4}$

Although, combined training methods consisting of heavy loads (>80\% 1RM) in conjunction with lighter loads performed ballistically have been reported to improve power; ${ }^{4}$ authors have also investigated the acute effects of combined training with lighter loads. Smilios and colleagues ${ }^{7}$ investigated the effect of contrast training with $30 \%$ 1RM half squat on bodyweight jump performance in trained regional-level team sport athletes. It was reported that loaded jump squats of 30\% 1RM produced significant improvements (4\%) in a subsequent bodyweight jump. ${ }^{7}$ Additionally, Baker ${ }^{8}$ reported similar improvements $(5 \%)$ in a jump squat that was preceded by a $60 \% 1 \mathrm{RM}$ jump squat in professional rugby league players. However, the chronic effect of heavier vs lighter contrast training in elite athletes has not been established. Previous research has determined the training effects of heavy vs light ballistic training (without contrast training). McBride and colleagues ${ }^{9}$ investigated the effects of 8 wk of heavy or light jump squat training on strength and power development. It was reported that the velocity of the movement, as controlled by the load, plays a key role in velocity-specific training adaptations; that is, the heavy group produced greater improvements in force output, while the light group had greater improvements in velocity. Interestingly, both groups significantly increased lower body strength. Whether chronic improvements can be made with lighter contrast training loads over a longer training period needs to be established.

Many professional athletes, including those playing rugby union taper training load during each competition week in an attempt to optimize physical preparation. This taper allows athletes to express themselves in a nonfatigued and primed state during the weekly competition/ game. High force, lower velocity training is normally performed at the beginning of each training week, while lighter, higher velocity training is performed in the latter stages of the week (typical of compound training). In addition, in an attempt to maximize training quality, athletes may also perform complex and contrast training as part of their resistance training programs. Although the effects of combined training have been relatively well established, the effects of combined training methods with different intensities (heavy vs light contrast training) performed with a weekly taper (heavy day and lighter day) requires further attention. Anecdotally, the current best practice is to lift with heavier contrast training loads.

Professional rugby union players perform a variety of different training modes concurrently within a training phase, that is, strength and power, speed, anaerobic and aerobic conditioning, along with a variety of rugby specific training (skills, team plays, technical and tactical sessions). However, much of the current strength and conditioning literature does not address the issue of how concurrent training may influence strength and power adaptations, with the application of research studies involving single-mode (eg, resistance training) are still applied to team sport athletes who perform concurrent training. Understanding the effects of different resistance training methods within a competition phase involving concurrent training will enhance programming and subsequent training adaptation, enabling athletes to be better prepared for weekly competition as occurs in many team sports. Therefore, the purpose of this investigation was to compare the effects of two contrast training programs on a range of lower body performance measures in highlevel rugby union players during the competition phase of their season. Each program included a tapering of loading (higher force early in the week, higher velocity later in the week), with the major difference between the two programs being the loading. Either a heavy (strengthpower) or a lighter (speed-power) resistance program was performed, which therefore affected the movement velocity that could be produced during each exercise set. It was hypothesized that the strength-power program would result in greater improvements in performance measures requiring higher force production (eg, weighted jumps), whereas the speed-power program would result in greater improvements in performance where high levels of velocity were required (eg, bodyweight jumps).

\section{Methods}

\section{Subjects}

Eighteen high-level rugby union players from a New Zealand provincial representative team (semiprofessional and professional players) volunteered to take part in this study (Table 1) during the final 2 wk of preseason training and the first $7 \mathrm{wk}$ of the competitive phase of the season. The intervention period included a $4 \mathrm{wk}$ baseline training and familiarization phase, during which time a lower-body maximal strength assessment (box squat) took place using methods previously described ${ }^{3,10}$ in order to characterize the training level of the subjects. Each player had at least $2 \mathrm{y}$ of resistance training experience and was deemed highly trained (see box squat strength, Table 1). Players were informed of the experimental risks and signed an informed consent document before the investigation. The investigation was approved by an institutional review board for use of human subjects.

\section{Table 1 Characteristics of high-level rugby union players in two separate training groups (mean \pm SD)}

\begin{tabular}{lcc}
\hline & $\begin{array}{c}\text { Strength-Power } \\
(\boldsymbol{n}=9)\end{array}$ & $\begin{array}{c}\text { Speed-Power } \\
(\boldsymbol{n}=9)\end{array}$ \\
\hline Age $(\mathrm{y})$ & $23 \pm 2$ & $25 \pm 2$ \\
Height $(\mathrm{cm})$ & $186 \pm 1$ & $187 \pm 1$ \\
Weight $(\mathrm{kg})$ & $99 \pm 10$ & $102 \pm 9$ \\
Box squat 1RM (kg) & $160 \pm 27$ & $176 \pm 17$ \\
\hline
\end{tabular}

Note. RM, repetition maximum. 


\section{Design}

Following a 4 wk baseline training and familiarization phase consisting of three resistance training sessions per week, players were assessed for peak power outputs during a bodyweight countermovement jump (BWCMJ), bodyweight squat jump (BWSJ), $50 \mathrm{~kg}$ countermovement jump (50CMJ), $50 \mathrm{~kg}$ squat jump (50SJ), depth jump (DJ), and broad jump (BJ). Players were then matched on playing position and BWCMJ power and were randomly allocated to either the strength-power or speed-power training group. Each group completed a 4 wk training intervention consisting of two training sessions per week and were then reassessed at the end of the training intervention. Power was assessed using the countermovement jump, squat jump, DJ and BJ exercises. These exercises were selected due to their common usage in power training programs and research studies and their ability to represent lower-body power. ${ }^{3,11-13}$ Additionally, these exercises were selected as they provide a "profile" of the specific areas of power production, that is, loaded and unloaded, inclusion or exclusion of stretch shortening cycle, vertical and horizontal axis, and tendon compliancy. ${ }^{14}$ Peak power was selected as the dependent measure as it has been reported to have the greatest association with athletic performance. ${ }^{15}$

\section{Methodology}

In order to characterize the training level of the subject, each player was assessed for maximal lower-body strength using the box squat exercise. Briefly, each player was required to perform three sets $(50 \%, 70 \%, 90 \%$ effort, two-six repetitions) of submaximal box squat followed by one set to failure of one to four repetitions. During the box squat, players used a self-selected foot position, and were required to lower themselves to a sitting position briefly on the box and then return to a standing position. The box height was adjusted for each player to allow the top of the thighs to be parallel to the floor while in the seated position. ${ }^{3,10}$ Three minutes rest was allowed between each set. Each set to failure was used to predict the players' one repetition maximum (1RM). ${ }^{16,17}$

Players performed two repetitions of BWCMJ, BWSJ, 50CMJ, 50SJ, DJ and BJ. Each jump was performed on a commercially available portable force plate (400 Series Performance Force Plate, Fitness Technology, Australia). For all jumps, no arm swing was allowed, the only exception being the BJ in which an arm swing was permitted. A position transducer (PT5A, Fitness Technology, Australia) was connected to a broomstick (vertical bodyweight jumps) or Olympic weightlifting bar (vertical weighted jumps) and was held across the posterior deltoids at the base of the neck. For the BWSJ and 50SJ players lowered themselves to approximately $90^{\circ}$ flexion of the knee, paused for $3 \mathrm{~s}$ and then jumped on the command "go." 14 The BWCMJ and 50CMJ were performed in the same manner with no pause between eccentric and concentric movements. The DJ consisted of participants standing on a box $30 \mathrm{~cm}$ above the force plate, stepping off the box and attempting to jump as quickly and as high as possible after foot contact (players were given the instructions to pretend that the force plate was "very hot" to minimize contact time on the force plate). The DJ score was determined by dividing the jump height by the contact time and will be referred to as the reactive strength index (RSI) from herein. ${ }^{18,19}$ The BJ was performed without the use of the force plate, and players were permitted the use of arm swing and were instructed to jump horizontally for maximal distance from a stationary position. Broad jump distance was measured as the distance from the front of the toes before take off, to the back off the heel on landing. The testing protocol was performed $7 \mathrm{~d}$ before the beginning of the first training session. All players had been familiarized with the testing battery before testing.

Both the force plate and position transducer were interfaced with computer software (Ballistic Measurement System, Fitness Technology, Australia) that allowed direct measurement of force-time characteristics (force plate) and displacement-time and velocity-time (position transducer) variables as outlined by Dugan and colleagues. ${ }^{15}$ The best value for each jump type was used for analysis.

\section{Training}

It has been previously reported that performance gains in a preseason training phase may essentially be a return to prior fitness levels. ${ }^{10}$ Therefore, as this investigation commenced during the preseason training phase, all players underwent a monitored $4 \mathrm{wk}$ base training phase to ensure that they were in a well-trained state before the beginning of the training intervention. The base training phase consisted of two 60 min rugby training sessions per week, three 45-60 min conditioning training sessions per week, one strength and plyometrics session (strength, three to four sets $\times 2-6 \mathrm{RM}, 3 \mathrm{~min}$ rest for four to six exercises; plyometrics, 3 sets $\times 4$ reps, 3 min rest for three exercises); one hypertrophy session (four sets $\times 8-12$ RM, 90 s rest for five exercises); and a circuit training session (6-12 reps, 30 s rest for 10 exercises, 30 min in duration). On the final week of the base training phase, all players were assessed for the maximum load that could be lifted for two to four repetitions in all the training exercises used in the intervention (except for sled sprint whereby a standardized load was used during the intervention phase ${ }^{20}$ ). The maximal two to four repetition testing allowed specific intensities and loads based on $1 \mathrm{RM}$ to be set for each individual during the intervention phase.

The intervention phase consisted of either a strength-power or speed-power resistance training performed twice a week for 4 wk during the competition phase of the season (Table 2). Each program included a tapering of loading (higher force early in the week, higher velocity later in the week). All the training sessions for the strength-power intervention were performed at a greater percent of 1RM than the speed-power inter- 
vention. For both interventions, exercises in the first training session were performed at a greater percent of $1 \mathrm{RM}$, while exercises in the second training session were performed at a lower percent of 1RM. The exercises in each training group (ie, strength-power and speed-power training) were matched for similar movement patterns, such as concentric focus, bilateral exercise. Therefore the major difference between each group was the load used, which based on the force-velocity relationship influenced the muscular forces and movement velocity that could be produced during the exercises. Players were instructed to perform all exercises as explosively as possible, with maximal intent.

\section{Additional Training}

In addition to the training described above, players also performed three upper body resistance exercises (85-95\% 1RM, three sets of four repetitions) during session 1 . During session 2 players performed two upper body resistance exercises in a ballistic fashion (40-60\% 1RM, three sets of four repetitions). Players also performed one speed development session with low resistance (20-30 min, including fast foot ladders, mini hurdles, maximal sprinting, over-speed sprinting), three team training sessions (30-75 min, including specific rugby skill, tactical, tackling, etc), one competitive match, and one recovery session (20-40 min, including light exercise, stretching, hot and cold baths) each week.

\section{Statistical Analysis}

All outcome measures_-peak power, reactive strength index and broad jump distance-are presented as mean \pm standard deviation. All data were log transformed to reduce nonuniformity of error, and the effects of the training phase were derived by back transformation as percent changes. ${ }^{21}$ Standardized changes in the mean of each measure were used to assess magnitudes of effects by dividing the changes by the between-player standard deviation. Standardized changes of $0.00-0.19 ; 0.20-0.59 ; 0.60-1.19$; and $\geq 1.20$ were interpreted as trivial, small, moderate, and large effects, respectively, ${ }^{22}$ a modification of Cohen's thresholds of $0.2,0.5$, and $0.8 .^{23}$ To make inferences about true (large-sample) value of an effect, the uncertainty in the effect was expressed as $90 \%$ confidence limits. The effect was deemed unclear if its confidence interval overlapped the thresholds for small positive and negative effects. ${ }^{23}$ To gain insight into the relative influence of the force and velocity components to the improvements in jump power, subsequent analysis of peak force and velocity data was then completed for measures that responded favorably to the training. Finally, correlational analysis was performed to assess the possibility of the difference in baseline strength affecting the magnitude of change in power. The kinetic and kinematic variables measured in this investigation have been shown to have good test-retest reliability $(R \leq 0.95 ; \mathrm{CV}<3.5 \%)$ when similar testing procedures were used with a comparable population. ${ }^{14,24}$

Table 2 Outline of lower body resistance training exercises in two separate lower body resistance training programs (strength-power and speed-power) in two groups of high-level rugby union players during a competition training phase

\begin{tabular}{lcccccc}
\hline & \multicolumn{2}{c}{ Session One } & & \multicolumn{2}{c}{ Session Two } \\
\cline { 2 - 3 } \cline { 6 - 7 } Exercise & Strength-Power Group & Speed-Power Group & & Strength-Power Group & Speed-Power Group \\
\hline $\mathbf{1}$ & Box squat (heavy) & Box squat (light) & & Jump squat (heavy) & Jump squat (light) \\
$\mathbf{2}$ & $10 \mathrm{~m}$ sled sprint $120 \mathrm{~kg}^{\#}$ & $10 \mathrm{~m}$ sled sprint $30 \mathrm{~kg}^{\#}$ & & $10 \mathrm{~m}$ sled sprint $30 \mathrm{~kg}^{\#}$ & $10 \mathrm{~m} \mathrm{sprint}^{\#}$ \\
$\mathbf{3}$ & Deadlift & $1 / 3$ Rack squat & & Power clean & $90^{\circ}$ Static jump* \\
$\mathbf{4}$ & $20 \mathrm{~kg}$ box jump & Assisted jump* & & High box depth jump* & Low box depth jump*
\end{tabular}

Week 1

Week 2

Week 3

Week 4

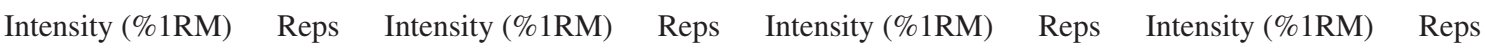

Strength-Power

\begin{tabular}{|c|c|c|c|c|c|c|c|c|}
\hline Session 1 & $80-90 \%$ & \multirow{2}{*}{$6,6,4,4$} & $90-95 \%$ & \multirow{2}{*}{$4,4,3,2$} & $95-98 \%$ & \multirow{2}{*}{$4,3,3,2$} & $90-95 \%$ & \multirow{2}{*}{$4,4,3,2$} \\
\hline Session 2 & $40-45 \%$ & & $45-50 \%$ & & $50-55 \%$ & & $45-50 \%$ & \\
\hline \multicolumn{9}{|l|}{ Speed-Power } \\
\hline Session 1 & $55-60 \%$ & \multirow{2}{*}{$6,6,4,4$} & $60-65 \%$ & \multirow{2}{*}{$4,4,3,2$} & $65-70 \%$ & \multirow{2}{*}{$4,3,3,2$} & $60-65 \%$ & \multirow{2}{*}{$4,4,3,2$} \\
\hline Session 2 & $20-25 \%$ & & $25-30 \%$ & & $30-35 \%$ & & $20-25 \%$ & \\
\hline
\end{tabular}

*Bodyweight exercise (repetitions 4,4,4); ${ }^{*}$ repetitions $1 \times 10 \mathrm{~m} \times 4$ sets. RM, repetition maximum. Exercises 1 and 2 , along with exercises 3 and 4 were performed using the contrast training method. 


\section{Results}

Baseline data for all measures are presented in Table 3. Both training groups were reasonably well matched for baseline scores with between-group differences reaching small magnitudes for the BWSJ and 50SJ only.

Inferences about the effect of each training program are shown separately (percent change) and comparatively (percent effect) in Table 4. There were smaller mean changes and larger standard deviations in the speed-power group for the 50CMJ, 50SJ, and RSI exercises which suggests that there were negative responders. Relative to the changes in the speed-power group, the strengthpower group produced small increases in 50CMJ (410 $\mathrm{W} ; 90 \%$ confidence limits, $\pm 380 \mathrm{~W}), 50 \mathrm{SJ}(360 ; \pm 480$ $\mathrm{W})$ and $\mathrm{BJ}(4 ; \pm 7 \mathrm{~cm})$. Alternatively, unclear betweengroup differences were observed in BWCMJ, BWSJ and RSI (Table 4).

Next, changes in peak force and velocity data were assessed in measures that responded favorably to training (ie, 50CMJ, 50SJ). Following the strength-power training, peak force improved by $12.1 \%( \pm 19 \%$; small $)$ and $26 \%$ $( \pm 22 \%$; large) in the 50CMJ and 50SJ, respectively. Only trivial improvements in peak force were observed for any of the measures in the speed-power group. A small increase in peak velocity was observed in the strengthpower group for the 50CMJ $(4.5 \pm 7.7 \%)$, while a small decrease in peak velocity occurred in the speed-power group in the $50 \mathrm{SJ}(-2.1 \pm 4.6 \%)$.

Correlations between baseline strength and the magnitude of the change in 50CMJ and 50SJ power ranged from $r=.17$ to $r=-.16$ suggesting that up to $3 \%$ of the variation in the change in power was due to differences in baseline strength. However, moderate correlations between baseline squat strength and change in 50CMJ were observed for force $(r=-.53)$ and velocity outputs during the 50CMJ ( $r=-.37$ ); suggesting that up to $29 \%$ and $14 \%$ of the change in force and velocity outputs could be explained by differing baseline strength levels.

Table 3 Baseline values (mean \pm SD) produced during different jumps in two separate groups of high-level rugby union players during a competition training phase

\begin{tabular}{lcc}
\hline & Strength-Power & Speed-Power \\
\hline BWCMJ (W) & $6560 \pm 820$ & $6740 \pm 930$ \\
BWSJ (W) & $6650 \pm 840$ & $6390 \pm 660$ \\
$50 \mathrm{CMJ}(\mathrm{W})$ & $5440 \pm 990$ & $5530 \pm 660$ \\
$50 S J(W)$ & $5280 \pm 920$ & $5050 \pm 490$ \\
RSI $\left(\mathrm{m} \cdot \mathrm{s}^{-1}\right)$ & $1.83 \pm 0.27$ & $1.86 \pm 0.30$ \\
BJ $(\mathrm{cm})$ & $252 \pm 22$ & $253 \pm 19$ \\
\hline
\end{tabular}

Note. BWCMJ, bodyweight countermovement jump; BWSJ, bodyweight static jump; 50CMJ, $50 \mathrm{~kg}$ countermovement jump; 50SJ, 50 kg static jump; RSI, reactive strength index; BJ, broad jump.
Only trivial correlations were observed between squat strength and change in 50SJ force and velocity. Finally, the correlation between baseline strength and change in BJ distance was $r=-.30$, explaining up to $9 \%$ of the variation of the change in $\mathrm{BJ}$.

In addition to maximal lower-body strength being assessed before the training program so that training intensities could be set, lower-body strength was also assessed by the conditioning coach in eight players from the strength-power program, and four players from the speed power program following the 4 wk training phase. A small increase of $4.8 \%( \pm 13 \%)$ was observed in the 12 players assessed. Athletes in the strength-power program increased strength by $3 \%( \pm 17 \%)$, while speed-power program athletes improved by $8 \%( \pm 3 \%)$.

\section{Discussion}

Findings from the current investigation suggested that the strength-power program was superior to the speedpower program, resulting in larger and more uniform improvements in various measures of lower body power. The strength-power program also successfully improved power in a greater number of performance measures, while the speed-power program only resulted in a small increase in a single measure. However, this single improvement for the speed-power program was less than that in the strength-power program.

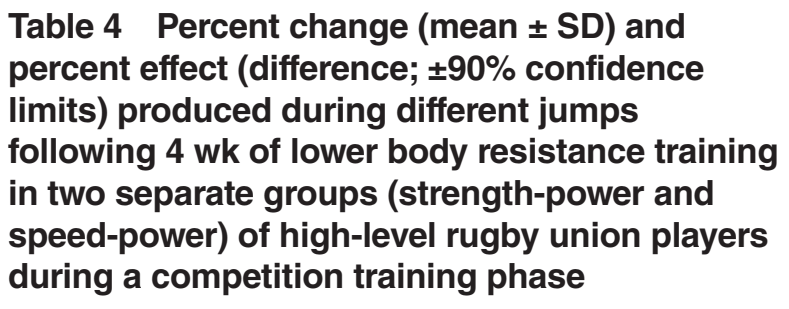

\begin{tabular}{|c|c|c|c|}
\hline & $\begin{array}{c}\text { Strength-Power } \\
(\%)\end{array}$ & $\begin{array}{c}\text { Speed-Power } \\
(\%)\end{array}$ & $\begin{array}{l}\text { Strength-Speed } \\
\text { Difference }^{*}(\%)\end{array}$ \\
\hline BWCMJ & $\begin{array}{c}1.6 \pm 3.1 \\
\text { trivial }\end{array}$ & $\begin{array}{c}0.8 \pm 3.4 \\
\text { trivial }\end{array}$ & $\begin{array}{c}0.8 \pm 4.3 \\
\text { unclear }\end{array}$ \\
\hline BWSJ & $\begin{array}{c}-1.4 \pm 4.2 \\
\text { trivial }\end{array}$ & $\begin{array}{c}0.4 \pm 4.0 \\
\text { unclear }\end{array}$ & $\begin{array}{c}-1.9 \pm 5.5 \\
\text { unclear }\end{array}$ \\
\hline $50 \mathrm{CMJ}$ & $\begin{array}{l}11.7 \pm 6.5 \\
\text { moderate }\end{array}$ & $\begin{array}{c}3.1 \pm 4.8 \\
\text { trivial }\end{array}$ & $\begin{array}{c}7.7 \pm 7.7 \\
\text { small }\end{array}$ \\
\hline $50 \mathrm{SJ}$ & $\begin{array}{l}11.2 \pm 5.6 \\
\text { moderate }\end{array}$ & $\begin{array}{l}4.4 \pm 9.6 \\
\text { unclear }\end{array}$ & $\begin{array}{c}6.9 \pm 9.7 \\
\text { small }\end{array}$ \\
\hline RSI & $\begin{array}{c}0.8 \pm 5.8 \\
\text { unclear }\end{array}$ & $\begin{array}{c}3.4 \pm 19.1 \\
\text { unclear }\end{array}$ & $\begin{array}{c}-2.6 \pm 22.8 \\
\text { unclear }\end{array}$ \\
\hline $\mathrm{BJ}$ & $\begin{array}{c}3.6 \pm 2.5 \\
\text { small }\end{array}$ & $\begin{array}{c}1.8 \pm 1.5 \\
\text { small }\end{array}$ & $\begin{array}{c}1.7 \pm 2.8 \\
\text { small }\end{array}$ \\
\hline
\end{tabular}

Note. BWCMJ, bodyweight countermovement jump; BWSJ, bodyweight static jump; 50CMJ, $50 \mathrm{~kg}$ countermovement jump; $50 \mathrm{SJ}$, $50 \mathrm{~kg}$ static jump; RSI, reactive strength index; BJ, broad jump. *Change in strengthpower group compared with change in speed-power group. 
Previous investigations examining changes in lower body power during a competitive season in the rugby codes have reported maintenance at best. ${ }^{2,3}$ Argus and colleagues $^{3}$ reported a small $3.3 \%$ decrease, while Baker ${ }^{2}$ reported a trivial $0.3 \%$ increase in weighted countermovement jump power. However, attempting comparisons between the current and previous investigations ${ }^{2,3}$ has several limitations. Firstly, the current investigation only consisted of a short phase at the start of a competitive season. Secondly, the specific detail of the resistance training programs used in the previous investigations was not fully reported. Future research should attempt to monitor changes over a longer competitive phase of the season using similar programming strategies to allow for more detailed comparisons. Nonetheless, the strength-power training program in the current investigation resulted in moderate improvements in both weighted countermovement jump power $(12 \%)$ and weighted squat jump power (11\%).

Strength-power training was superior to the speedpower training program resulting in larger improvements in a greater number of measures of jump performance. In contrast, McBride and colleagues ${ }^{9}$ who investigated the effects of training with heavy (80\% 1RM) or light $(30 \%$ $1 \mathrm{RM}$ ) jump squats reported that light jump squat training improved performance in a greater number of measures than heavy jump squat training. Harris and colleagues ${ }^{4}$ reported improvements in a greater number of performance measures following a high power training program when compared with a high force program. Although in both investigations ${ }^{4,9}$ the higher load group improved to a greater extent in high force output measures (1RM values), whereas the lower load group showed the greatest improvement in higher velocity-related movements. Differences in methodology, including the length of the intervention period and utilization of the contrast training method may help to explain some of the variation between the current investigation and previous literature..$^{4,9}$

Although similar exercises, sets and repetitions were performed by the two groups, the current investigation did not match training volume. As such, unequal resistance training volume between the strength-power and speed-power groups may have been partially responsible for the differences observed. Although the resistance training volume performed cannot be easily determined post training due to some of the exercises performed (eg, sled sprints and bodyweight exercises), and force outputs or repetition contraction time not measured during training; it is likely that the strength-power group performed a greater training volume. Indeed Crewther and colleagues ${ }^{25}$ reported that when repetitions are performed with maximal intent, as in the current study, an increase in load of $10 \%$ results in a $14 \%$ increase in time under tension (TUT) and $15 \%$ increase in work done. The participants in the strength power group performed on average $25 \% 1 \mathrm{RM}$ greater intensity than the speed-power group during the four week intervention (although bodyweight exercises and sled pulls could not be accounted for in this calculation). Therefore the greater intensity performed in the strength-power group may have resulted in approximately $35 \%$ greater TUT and $38 \%$ more work done and may be the differentiating factor between the two training programs. In the investigation by McBride and colleagues ${ }^{9}$ discussed above, participants in the light jump squat group performed an additional set of jumps in an attempt to equate overall workloads over the training period. The equal-volume training load may help to explain the performance improvements observed by McBride and colleagues ${ }^{9}$ in both the heavy and light jump squat training groups

The greatest improvement in performance measures for the present study were observed in the weighted jumps. Tuomi and colleagues ${ }^{26}$ suggested that initial performance adaptations during combined training methods have a greater effect on higher force rather than lower force producing activities. In addition, previous authors have reported that heavier resistance training results in greater improvements to the higher end of the forcevelocity curve while lighter resistance training result in improvements in the lower end. ${ }^{4,9}$ Training intensities for the strength-power program in session one ranged from $80 \%$ to $98 \%$ 1RM which emphasizes the higher end of the force-velocity curve. The strength-power program also trained with intensities ranging from 45-55\% 1RM during session two which was slightly heavier than the testing weight. It is likely that the higher training load performed by the strength-power group resulted in a greater adaptation in the weighted jumps due to the greater volume of training performed at similar resistances. Attempting to move large external loads may induce a number of adaptations including an increase in contractile force which may be realized through increased neural activation, reduced coactivation as well as a number of muscle architectural or fiber size adaptations. $5,27-29$ Therefore, training with greater resistance regularly, as in the strength-power program, may have provided an increased neuromuscular stimulus resulting in greater performance benefits. Likewise, the lack of improvement in the weighted jump measures in the speed-power program may have been due to inadequate exposure to higher loads. The speed-power program only trained with moderate to heavy loads ( $55 \%$ to $70 \% 1 \mathrm{RM})$ once a week, while the second session was performed using loads from bodyweight to $35 \%$ 1RM. As such, training with only one heavier stimulus each week appears to be inadequate for performance improvements in measures which require higher force production.

Similarly, the lack of improvement in the bodyweight jumps (excluding broad jump) in both programs may have been due to the insufficient total volume or stimulus of the jump training performed. It has been suggested that improvements in activities requiring greater velocity (ie, bodyweight or low resistance plyometrics) may need a longer training period or greater training volume for adaptations to present. ${ }^{12,26}$ In a recent meta-analysis, de Villarreal and colleagues ${ }^{12}$ reported that training volumes of more than $10 \mathrm{wk}$ maximize the probability of obtaining significantly greater improvements in bodyweight vertical jump performance. De Villarreal and colleagues ${ }^{12}$ reported 
that for optimal improvements in bodyweight vertical jump performance, training programs should include 50 contacts twice a week (100 total). In the current investigation, neither program performed 100 contacts per week. The strength-power program performed between 38-52 contacts each week while the speed-power program performed between 49-56 contacts per week. It appears that the total volume of contacts may have been inadequate to produce improvements in bodyweight vertical jump performance. The volume of contacts performed in the current study was limited by the players' strength and conditioning coach. The players were not accustomed to performing 100 jump contacts within their resistance training sessions, and it was deemed that the increased jump volume may have had potential for injury. All jump exercises had been regularly performed by the players in a contrast or complex training method within their normal training programs for at least 12 mo before the investigation. As such, the continual performance of the bodyweight jump without any significant increases in intensity or training stress would likely have only maintained performance.

The athletes in the current investigation performed resistance training in addition to several different training modes. Power development may be compromised by higher volumes of training performed (ie, during concurrent training); where as high force development may be less affected. ${ }^{2,30}$ Indeed, in two separate investigations Argus and colleagues ${ }^{3,10}$ reported that power development was more affected than strength (high force) development during a preseason and in-season training phase where concurrent training was performed. Although the $50 \mathrm{~kg}$ jumps performed in the current investigation were not a strength task; jumping with heavier loads produces greater force output than with lighter loads, such as bodyweight. ${ }^{31}$ Based on previous findings it may be speculated that the higher force producing weighted jumps may have been less affected by the higher volume of concurrent training performed. Therefore, the current investigations intervention period and contact volume may not have been an adequate stimulus for improvements to be made in bodyweight vertical jump measures. In addition, the concurrent training performed by the participants may have affected the higher velocity (bodyweight) jumps more so than the higher force producing weighted jumps.

The speed-power program resulted in smaller mean changes with larger standard deviation for the 50CMJ, 50SJ exercises and the RSI. These findings suggest that some individuals actually had performance decrements over the 4 wk training period. There were no similarities in baseline characteristics (eg, high power output) between the responders and nonresponders to explain the variability in the change of performance to the same training program. One mechanism proposed by Beaven and colleagues ${ }^{32}$ suggested that players have differing individual hormonal responses to a single resistance training session. In addition, when players trained using resistance training that elicited the greatest testosterone response, significant improvements in strength occurred. Conversely, when players trained using resistance training that produced the smallest testosterone response, $75 \%$ of players showed either no change or a significant decline in $1 \mathrm{RM}$ performance. ${ }^{33}$ Further research is still required to determine individual response to a training program.

Both programs produced small improvements in broad jump distance. Interestingly, neither of the programs included any jumps in the horizontal plane, the only possible exceptions being the weighted sled sprints. The players in the current investigation had traditionally performed vertically dominated plyometric training, and thus minimal horizontal plyometric training before this investigation. The small amount of horizontal training (weighted sled sprints) performed by the two programs may have been adequate to elicit improvement in broad jump distance due to the relativity unfamiliar stimulus. In conjunction with the weighted sled training, transference of training adaptation from horizontal training performed during the players' additional rugby trainings (eg, scrimmaging, mauling) may have also provided stimulus for adaption to occur. Indeed, if there had been a greater focus on horizontal power within the program there may have been greater increases in the broad jump for both groups and a potential between-group difference in response.

Although it has been suggested that the ability to develop high levels of muscular power is critical for successful performance in many sports, ${ }^{4}$ maximal strength is also important in most contact sports. ${ }^{2}$ For most athletes and conditioning coaches, improving maximal strength will be one of the performance goal priorities of the program. As such it should be noted that maximal box squat strength was assessed by the player's strength coach before and following the intervention phase in a total of 12 of the players participating in this investigation (eight strength-power, four speed-power). A small increase of $4.8 \%$; $( \pm 13 \%)$ was observed in the 12 players assessed. Athletes in the strength-power program increased strength by $3 \%( \pm 17 \%)$, while speed-power program athletes improved by $8 \%( \pm 3 \%)$.

\section{Practical Applications}

Performing heavy combined training twice a week is an effective method for improving a range of jump performance measures in high-level rugby union players over a 4 wk competitive phase. Our findings suggest that improvements in jump performance can be made in team sport athletes during the competitive season when athletes are exposed to higher volume-load stimuli which includes one heavy lifting session each week. Indeed, the use of heavier resistance combined training (strength-power) produced larger improvements in a greater number of performance measures than similar programming performed with lighter resistances. For practitioners and athletes who regularly compete once a week during the competition phase, the use of high force combined training consisting of contrast training with a heavy day and lighter day is an effective way to make improvements in performance over a short training phase during the competitive season. Finally, a greater volume of lower resistance plyometric 
training may be required for athletes to enhance vertical bodyweight jump performance.

\section{References}

1. Baker D. Differences in strength and power among juniorhigh, senior-high, college-aged, and elite professional rugby league players. J Strength Cond Res. 2002;16:581-585.

2. Baker D. The effects of an in-season of concurrent training on the maintenance of maximal strength and power in professional and college-aged rugby league football players. J Strength Cond Res. 2001;15:172-177.

3. Argus CK, Gill ND, Keogh JWL, Hopkins WG, Beaven CM. Changes in strength, power and steroid hormones during a professional rugby union competition. J Strength Cond Res. 2009;23:1583-1592.

4. Harris GR, Stone MH, O’Bryant HS, Proulx CM, Johnson RL. Short-term performance effects on high power, high force, or combined weight-training methods. $J$ Strength Cond Res. 2000;14:14-20.

5. Mangine GT, Ratamess NA, Hoffman JR, Faigenbaum AD, Kang J, Chilakos A. The effects of combined ballistic and heavy resistance training on maximal lower- and upper-body strength in recreationally trained men. J Strength Cond Res. 2008;22:132-139.

6. Duthie GM, Young WB, Aitken DA. The acute effects of heavy loads on jump squat performance: An evaluation of the complex and contrast methods of power development. J Strength Cond Res. 2002;16:530-538.

7. Smilios I, Pilianidis T, Sotiropoulos K, Antonakis M, Tokmakidis SP. Short-term effects of selected exercise and load in contrast training on vertical jump performance. J Strength Cond Res. 2005;19:135-139.

8. Baker D. A series of studies on the training of high-intensity muscle power in rugby league football players. J Strength Cond Res. 2001;15:198-209.

9. McBride JM, Triplett-McBride T, Davie A, Newton RU. The effect of heavy- vs. light-load jump squats on the development of strength, power, and speed. J Strength Cond Res. 2002;16:75-82.

10. Argus CK, Gill N, Keogh J, Hopkins WG, Beaven CM. Effects of a short-term pre-season training programme on the body composition and anaerobic performance of professional rugby union players. J Sports Sci. 2010;28:679-686.

11. Baker D, Nance S, Moore M. The load that maximizes the average mechanical power output during jump squats in power-trained athletes. J Strength Cond Res. 2001;15:92-97.

12. De Villarreal ESZ-S, Kellis E, Kraemer WJ, Izquierdo M. Determining variables of plyometric training for improving vertical jump height performance: a meta-analysis. $J$ Strength Cond Res. 2009;23:495-506.

13. Bissas AI, Havenetidis K. The use of various strength-power tests as predictors of sprint running performance. $J$ Sports Med Phys Fitness. 2008;48:49-54.

14. McGuigan MR, Cormack S, Newton RU. Long-term power performance of elite Australian rules football players. $J$ Strength Cond Res. 2009;23:26-32.

15. Dugan EL, Doyle TLA, Humphries B, Hasson CJ, Newton RU. Determining the optimal load for jump squats: A review of methods and calculations. J Strength Cond Res. 2004;18:668-674.
16. Lander J. Maximums based on reps. Natl Str Cond Assoc. 1985;6:60-61.

17. LeSuer DA, McCormick JH, Mayhew JL, Wasserstein RL, Arnold MD. The accuracy of prediction equations for estimating 1-RM performance in the bench press, squat, and deadlift. J Strength Cond Res. 1997;11:211-213.

18. Ebben WP, Petushek EJ. Using the reactive strength index modified to evaluate plyometric performance. J Strength Cond Res. 2010;24:1983-1987.

19. Flanagan EP, Comyns TM. The use of contact time and the reactive strength index to optimize fast stretch-shortening cycle training. Strength Condit J. 2008;30:32-38.

20. Keogh JWL, Newlands C, Blewett S, Chun E-L, Payne A. A kinematic analysis of a strongman-type event: the heavy sprint-style sled pull. J Strength Cond Res. 2010;24:3088-3097.

21. Hopkins WG. Spreadsheets for analysis of controlled trials with adjustment for a predictor. Sportscience 2006;10 (sportsci.org/2006/wghcontrial.htm).

22. Snowling NJ, Hopkins WG. Effects of different modes of exercise training on glucose control and risk factors for complications in type 2 diabetic patients: a meta-analysis. Diabetes Care. 2006;29:2518-2527.

23. Batterham AM, Hopkins WG. Making meaningful inferences about magnitudes. Int J Sports Physiol Perform. 2006;1:50-57.

24. Cormack SJ, Newton RU, McGuigan MR, Doyle TLA. Reliability of measures obtained during single and repeated countermovement jumps. Int $J$ Sports Physiol Perform. 2008;3:131-144.

25. Crewther B, Cronin J, Keogh J. Possible stimuli for strength and power adaptation: Acute metabolic responses. Sports Med. 2006;36:65-78.

26. Toumi H, Best TM, Martin A, Poumarat G. Muscle plasticity after weight and combined (weight + jump) training. Med Sci Sports Exerc. 2004;36:1580-1588.

27. Cronin J, McNair PJ, Marshall RN. The effects of bungy weight training on muscle function and functional performance. J Sports Sci. 2003;21:59.

28. Blazevich AJ, Gill ND, Bronks R, Newton RU. Trainingspecific muscle architecture adaptation after 5-wk training in athletes. Med Sci Sports Exerc. 2003;35:2013-2022.

29. Newton RU, Kraemer WJ, Hakkinen K. Effects of ballistic training on preseason preparation of elite volleyball players. Med Sci Sports Exerc. 1999;31:323-330.

30. Kraemer WJ, Patton JF, Gordon SE, et al. Compatibility of high-intensity strength and endurance training on hormonal and skeletal muscle adaptations. J Appl Physiol. 1995;78:976-989.

31. Crewther B, Cronin J. A comparison of heavy and light load training and their proposed effects on increasing strength and hypertrophy. Strength \& Conditioning Coach. 2002;10:15-18.

32. Beaven CM, Gill ND, Cook CJ. Salivary testosterone and cortisol responses in professional rugby players after four resistance exercise protocols. J Strength Cond Res. 2008;22:426-432.

33. Beaven CM, Cook CJ, Gill ND. Significant strength gains observed in rugby players after specific resistance exercise protocols based on individual salivary testosterone responses. J Strength Cond Res. 2008;22:419-425. 
Copyright of International Journal of Sports Physiology \& Performance is the property of Human Kinetics Publishers, Inc. and its content may not be copied or emailed to multiple sites or posted to a listserv without the copyright holder's express written permission. However, users may print, download, or email articles for individual use. 